\title{
Dressed-atom approach to embedding and physisorption in metals
}

\author{
A. N. Andriotis and C. A. Nicolaides \\ Theoretical and Physical Chemistry Institute, National Hellenic Research Foundation, \\ 48 Vassileos Constantinou Avenue, 11635 Athens, Greece
}

(Received 28 July 1986)

\begin{abstract}
A number of solid-state situations exhibit mainly atomic characteristics. Accordingly, for phenomena involving metals, we have developed a "dressed-atom" approach which is implemented computationally via a coupled Hartree-Fock procedure, and which incorporates the surrounding metallic character self-consistently into the dressed-ground-state or excited-atomic-state wave function. The systems which are studied here are $\mathrm{He} / \mathrm{Na}(001), \mathrm{He} / \mathrm{Al}(001)$, and $\mathrm{Ar} / \mathrm{Na}(001)$. The correlation-energy contribution to the self-consistent metal-adatom interaction is considered in the image-potential approximation. It is found that (i) argon binds on $\mathrm{Na}(001)$ with an energy of about $60 \mathrm{meV}$, and (ii) it is more likely for $\mathrm{Ar} / \mathrm{Na}(001)$ to exhibit its ionic rather than its neutral configuration during excitation from the ground ${ }^{1} S$ state.
\end{abstract}

\section{INTRODUCTION}

The studies which are reported in the present paper are based on the following assumption: For many solid systems and for many spectroscopic phenomena, a good description of the physical state and/or process can be obtained by focusing not on the solid as a whole but on a concept of a dressed atom (DA), i.e., an atom whose wave functions and properties are perturbed by the surrounding solid-state medium. The computational implementation of this concept puts emphasis on the accurate description of the isolated ground or excited atomic state and then on its perturbation by the medium. ${ }^{1-6}$ The perturbation is taken to be the result of a self-consistent interaction between the atom and the medium. The gross (zeroth-order) description of electronic structure is done at the HartreeFock level-especially for closed-shell systems. Once the form of the perturbation is established from a reliable theoretical model, its effect on the DA wave function is taken into account by a self-consistent computation of perturbed orbitals, in analogy with the coupled HartreeFock theory (CHF), of induced moments due to external electric or magnetic fields. ${ }^{7-9}$

The DA approach to aspects of the physics of the solid state can account for atomic electron correlation and relativistic effects in ground and excited states systematically and accurately. This constitutes a definite advantage over the standard solid-state approaches. On the other hand, being an approximation to the solid state it makes sense only when the experimental probing is such that the atomic characteristics and effects on the phenomenon are expected to remain strong. For example, the whole field of spectroscopy of inner electrons of chemisorbed or bulk atoms can be understood to a high degree of accuracy in this way. ${ }^{1-5,9}$ Similarly, even cases of optical spectroscopy of the solid state can, in principle, be tackled, whenever there is evidence of enhanced atomic character. ${ }^{10-15}$ In fact, the present work is inspired by the second example, which concerns the field of adsorbed noble gases on metals. ${ }^{10-15}$
Regarding the degree of approximation in describing the interaction between the atom and the medium, the present approach may be considered as a generalization of recent theoretical approaches to embedding or physisorption problems. In particular, it has been applied to the case of physisorption of helium atoms on metal surfaces. ${ }^{16,17}$ These applications, within our CHF calculation scheme, allowed a study of the factors which affect the linear relationship ${ }^{18}$ between the interaction energy of a helium atom and the free-electron density of the metal with which the helium atom interacts.

In the present work we have studied the following.

(1) The interaction of helium with the $\mathrm{Na}(001)$ and $\mathrm{Al}(001)$ semi-infinite metals.

(2) The interaction of ground $K L 3 s^{2} 3 p^{6}{ }^{1} S$ and excited $\left(K L 3 s^{2} 3 p^{5} 4 s^{3} P^{o}\right)$ argon and of singly ionized $\mathrm{Ar}^{+}(K L$ $\left.3 s^{2} 3 p^{52} P^{o}\right)$ with the $\mathrm{Na}(001)$ semi-infinite metal. [Due to the fact that the ${ }^{3} P^{o}$ Hartree-Fock function of the excited argon $\left(K L 3 s^{2} 3 p^{5} 4 s\right)$ is practically identical to the ${ }^{1} P^{o}$ one-their Hartree-Fock energies differ only by $0.17 \mathrm{eV}$ and the average radii of the $4 s$ orbital differ only by $2 \%$-we have employed the triplet state for convergence reasons and computational efficiency.]

\section{COUPLED HARTREE-FOCK THEORY FOR A NOBLE GAS PHYSISORBED ON A METAL SURFACE}

Our DA approach first separates the problem into three parts.

(i) The isolated atom in its ground or excited state.

(ii) The surrounding medium-be it the surface or the bulk.

(iii) The self-consistent interaction between the atom and the medium.

The calculation is carried out in the following way.

(I) The correlated atomic wave functions and properties are obtained accurately from the state-specific theory of atomic structure as developed and applied by Beck and 
Nicolaides. ${ }^{1,19,20}$ The reader is referred to these papers for the presentation of the theory. One of its semiquantitative results is the possibility of identifying a priori the most important correlation effects which influence the electronic spectra of atoms and solids.

(II) The surrounding medium is taken to be a semiinfinite metal occupying the negative $z$ space. Its surface lattice plane is located at $z=0.0$ and it exhibits the $\langle h k l\rangle$ orientation. The metal substrate is treated according to a generalized jellium approach which is described elsewhere. $^{21,22}$ This approach has the advantage of incorporating part of the lattice effects, thus allowing the specification of the crystal orientation $\langle h k l\rangle$. On the other hand, it retains the simplicity of the usual jellium model. In particular, the periodicity along the negative $z$ axis is considered explicitly but invariance is assumed along the $x y$ planes. For this system, the single-electron wave functions have the form

$$
\psi_{\mathbf{k}}(\mathbf{r})=\left(\frac{2}{\Omega}\right)^{1 / 2} e^{i \mathbf{k}_{\| \mid} \cdot \boldsymbol{\rho}_{\xi}(z)},
$$

where $\Omega$ is the volume of the system, and the notation $\mathbf{r}=(\boldsymbol{\rho}, z), \mathbf{k}=\left(\mathbf{k}_{\|}, q\right)$ is used, which implies that $\mathbf{k}_{\|}$and $\boldsymbol{\rho}$ are vectors parallel to the metal surface. Although $q$ is determined from the branch of the band structure which is specified by the $\langle h k l\rangle$ orientation, it is assumed that the maximum (Fermi-level) kinetic energy of the metallic electrons, $E_{F}$, is given by

$$
2 E_{F}=k_{F}^{2} \text {. }
$$

The single-electron wave functions $\xi_{q}(z)$ and the singleelectron potential $V_{\text {eff }}(z)$ for the metal substrate are obtained by solving Schrödinger's equation selfconsistently. ${ }^{21}$

(III) The interaction between the atom and the surrounding medium is described by $V(\mathbf{r})$, which includes the following.

(i) The Coulomb term, $V_{\text {Coulomb }}(\mathbf{r})$, which is the Coulombic contribution to the single-electron potential $V_{\text {eff }}$ of the metal substrate.

(ii) The exchange interaction $V_{x}(\mathbf{r})$ between the electrons of the substrate and those of the adatom, i.e.,

$$
V_{x}(\mathbf{r}) \psi_{a}(\mathbf{r})=-\frac{1}{2} \sum_{\mathbf{k}} \int \frac{\psi_{\mathbf{k}}(\mathbf{r}) \psi_{\mathbf{k}}^{*}\left(\mathbf{r}^{\prime}\right) \psi_{a}\left(\mathbf{r}^{\prime}\right)}{\left|\mathbf{r}-\mathbf{r}^{\prime}\right|} d \mathbf{r}^{\prime},
$$

where $\psi_{a}(\mathbf{r})$ denotes the single-electron orbitals of the adatom, and the $\mathbf{k}$ summation includes the spin indices also.

(iii) The correlation potential $V_{\text {corr }}(\mathbf{r})$. In the present calculations, this is approximated by the image potential between the adatom and its own image with respect to the image plane located at $z=z_{\mathrm{im}}$. In particular, we take $\mathrm{e}^{23}$

$$
V_{\text {corr }}\left(z_{0}\right)=-\sum_{i}\left(\frac{z_{i}^{2}+\frac{1}{2} \rho_{i}^{2}}{8\left(z_{0}-z_{\mathrm{im}}\right)^{3}}\right),
$$

where $\mathbf{r}_{i}=\left(\rho_{i}, z_{i}\right)$ is the position vector of the $i$ th electron of the adatom, whose nucleus is located at $z=z_{0}$.

The form of $V_{\text {corr }}(\mathbf{r})$ given by Eq. (4) is based on the assumption that each electron of the adatom "sees" only its own image and that the effect of the interaction between the image charges can be neglected. ${ }^{23}$ Another assumption which is implied by Eq. (4) is that only the leading (van der Waals) term is retained in the expansion in inverse powers of $z_{0}-z_{\text {im }}$ of the interaction between a dipole at $z_{0}$ and its own image with respect to the image plane at $z=z_{\text {im }}$.

The form of the correlation contribution to the interaction between the adatom and the metal surface which is given by Eq. (4) has the disadvantage that it is singular for $z_{0}=z_{\text {im }}$. However, this disadvantage can be eliminated by taking the saturated form of the correlation potential, which has the asymptotic form $-1 / 4\left(z_{0}-z_{\mathrm{im}}\right)$ at large metal-adatom separations and tends to a saturation value as the adatom approaches the metallic surface. ${ }^{24,25}$ It is only for computational reasons that we have kept the form given by Eq. (4) in the present calculations. This approximation is well justified at small-overlap regions. In order to overcome the difficulty of defining the position $z_{\text {im }}$ of the image plane, ${ }^{26}$ we have chosen $z_{\text {im }}$ so that the potential $V_{\text {corr }}(z)$ for a helium atom interacting with a metal surface has approximately the same value as the correlation potential

$$
E_{\mathrm{vdW}}(z)=-\frac{C_{\mathrm{ZK}}}{\left(z-z_{\mathrm{ZK}}\right)^{3}},
$$

whose parameters $C_{\mathrm{ZK}}$ and $z_{\mathrm{ZK}}$ were given by Zaremba and $\mathrm{Kohn}^{27}$ (or more recently by Persson and Zaremba ${ }^{28}$ ).

Given the several contributions to $V(\mathbf{r})$, we take

$$
V(\mathbf{r})=V_{\text {Coulomb }}(\mathbf{r})+V_{x}(\mathbf{r})+V_{\text {corr }}(\mathbf{r})
$$

as the external potential to the Hartree-Fock Hamiltonian of the free adatom. Then, the perturbed Hamiltonian of the adatom is solved self-consistently. The approach is analogous to the coupled Hartree-Fock approximation (CHF) for atoms and molecules in electromagnetic fields ${ }^{7}$ and, for the solid state, it has the advantage that it allows, in principle, the study of distortions of the ground as well as of the excited states of physisorbed atoms or ions to all orders-within the independent-particle model. The steps followed in obtaining the CHF solution can be summarized as follows.

The potential $V(\mathbf{r})$ is added to the atomic Hartree-Fock potential of the adatom and the resulting equations are solved self-consistently at each distance $z$.

In proceeding with the self-consistent solution, the single-electron atomic orbitals $\psi_{a}(\mathbf{r})$ are expanded in a series of Gaussian-type orbitals (GTO's) $g_{l_{i}}(r)$, i.e.,

$$
g_{l_{i}}(r)=c_{i} r^{n_{i}-1} e^{-\beta_{i} r^{2}}
$$

where $n_{i}=l_{i}+1$ and $c_{i}$ is a coefficient determined by solving the coupled Hartree-Fock Hamiltonian of the adatom. For the case of the ground state of the adatom the exponents $\beta_{i}$ are taken from the literature. However, for the excited cases, we had to enlarge the GTO basis set by including one or two diffuse GTO's. The coefficients were obtained by an energy-optimization procedure using the analytic Hartree-Fock computational technique of Roothaan. $^{29}$ 
The incorporation of the external interaction $V(\mathbf{r})$ into the integro-differential Hartree-Fock equations for the adatom $^{29}$ requires the calculation of the interaction integrals

$$
V_{i j}^{|m|}=\left\langle g_{l_{i}} Y_{l_{i}|m|}|V(\mathbf{r})| g_{l_{j}} Y_{l_{j}|m|}\right\rangle
$$

which are associated with $V(\mathbf{r})$. We have three types of such integrals. The integrals which are associated with the correlation potential $V_{\text {corr }}(\mathbf{r})$ can be easily obtained analytically.

The Coulomb integrals $J_{i j}^{|m|}$ associated with the Coulombic contribution $V_{\text {Coulomb }}(\mathbf{r})$, i.e.,

$$
J_{i j}^{|m|}=\int_{0}^{\infty} d r r^{2} g_{l_{i}}(r) g_{l_{j}}(r) \int_{0}^{2 \pi} d \phi \int_{0}^{\pi} d \vartheta \sin \vartheta V_{\text {Coulomb }}\left(r \cos \vartheta-z_{0}\right) Y_{l_{i}|m|}^{*}(\vartheta, \phi) Y_{l_{j}|m|}(\vartheta, \phi),
$$

are obtained by numerical integration.

Finally, the calculation of the exchange integrals $E_{i j}{ }^{m} \mid$, i.e.,

$$
E_{i j}^{|m|}=\left\langle g_{l_{i}} Y_{l_{i}|m|}|V(\mathbf{r})| g_{l_{i}} Y_{l_{j}|m|}\right\rangle=-\frac{1}{2} \sum_{k} \iint \frac{\psi_{\mathbf{k}}^{*}\left(\mathbf{r}^{\prime}\right) g_{l_{i}}\left(r^{\prime}\right) Y_{l_{i}|m|}\left(\vartheta^{\prime}, \phi^{\prime}\right) g_{l_{j}}^{*}(r) Y_{l_{j}|m|}^{*}(\vartheta, \phi) \psi_{\mathbf{k}}(\mathbf{r})}{\left|\mathbf{r}-\mathbf{r}^{\prime}\right|} d \mathbf{r} d \mathbf{r}^{\prime}
$$

proceeds by first performing the integration over the $\mathbf{k}_{\|}$component of the wave vector $\mathbf{k}$. This yields

$$
\begin{array}{r}
E_{i j}^{|m|}=\frac{a N_{i}^{(0)} N_{j}^{(0)}}{\pi} \sum_{q} \int d z_{1} \int d z_{2} \xi_{q}\left(z_{1}\right) \xi_{q}^{*}\left(z_{2}\right) \int d \rho_{1} \int d \rho_{2} r_{1}^{n_{i}-1} r_{2}^{n_{j}-1}\left(k_{F}^{2}-q^{2}\right)^{1 / 2} e^{-\beta_{i} r_{1}^{2}} e^{-\beta_{j} r_{2}^{2}} Y_{l_{i}|m|}\left(\vartheta_{1}, \phi_{1}\right) \\
\times Y_{l_{j}|m|}^{*}\left(\vartheta_{2}, \phi_{2}\right) \frac{J_{1}\left[\left|\boldsymbol{\rho}_{1}-\boldsymbol{\rho}_{2}\right|\left(k_{F}^{2}-q^{2}\right)^{1 / 2}\right]}{\left|\mathbf{r}_{1}-\mathbf{r}_{2}\right|\left|\boldsymbol{\rho}_{1}-\boldsymbol{\rho}_{2}\right|}
\end{array}
$$

where $a$ is the area of the metal surface, and $J_{1}(x)$ is the Bessel function of the first order. At this point we make a numerical approximation. We assume that $\left|\mathbf{r}_{i}-\mathbf{r}_{j}\right| \cong\left(\boldsymbol{\rho}_{i}-\boldsymbol{\rho}_{j} \mid\right.$ in the denominator of Eq. (10). Then, some of the integrations in Eq. (10) may be performed analytically and the rest are obtained numerically. This approximation may overestimate the pole at $\rho=\rho^{\prime}$ of the exchange integrals for short metal-adatom separations and in cases where diffusive Gaussian basis functions are employed to describe the adatom orbitals. However, at metal-adatom separations where the free-electron density of the metal substrate is low, compared to its bulk value, our calculations indicated that the approximation we have employed is satisfactory and has general application in the small-overlap region. Thus, using this approximation, the exchange integrals which involve $s, p_{x}, p_{y}$, and $p_{z}$ types of Gaussian basis functions take a form suitable for numerical integration.

Having obtained the one-electron integrals $V_{i j}^{|m|}$ we proceed with the self-consistent-field (SCF) solution in the Hartree-Fock approximation. For this purpose, we have generalized, as necessary, existing codes of the POLYATOM computer program. ${ }^{30}$

\section{THE METAL-ADATOM INTERACTIONS WITHIN THE CHF APPROXIMATION}

Previous theoretical studies on the interaction $E(\mathbf{r})$, of the noble-gas atom in its ground state with the metal substrates, ${ }^{27,31}$ have anticipated that this interaction can be decomposed into two contributions. One, $E_{\text {rep }}(\mathbf{r})$, is repulsive and varies more or less linearly with the unperturbed free-electron density, $\rho(\mathbf{r})$, of the metal substrate, i.e.,

$$
E_{\text {rep }}(\mathbf{r})=\alpha_{\text {eff } \rho(\mathbf{r}) .}
$$

The other, $E_{\mathrm{vdW}}(\mathbf{r})$, is attractive and is mainly due to the van der Waals interaction (correlation term) between the metal and the adatom. Equation (11) is the generalization of the result concerning the energy change upon embedding a noble-gas atom into a jellium-like metal. ${ }^{18,32-35}$ Recently, it was shown ${ }^{36}$ that, in the case of embedding, Eq. (11) constitutes the zeroth-order term, if $E_{\text {rep }}(\mathbf{r})$ is expressed as a power series in $\rho_{0}$, the bulk electron density of the metal, i.e., $\rho_{0}=k_{F}^{3} / 3 \pi^{2}$. In the case of the interaction of a helium atom with a metal surface, it was also shown ${ }^{17}$ that $\alpha_{\text {eff }}$ can be approximated analytically and that $\alpha_{\text {eff }}$ depends on the characteristics of both the metal substrate and the helium atom. The two terms $E_{\text {rep }}(\mathbf{r})$ and $E_{\mathrm{vdW}}(\mathbf{r})$ constituting $E(\mathbf{r})$ have been treated independently. In particular, Zaremba and Kohn ${ }^{31}$ showed that

$$
E_{\text {rep }}\left(\mathbf{r}_{0}\right)=\sum_{\mathbf{k}}\left[\varepsilon_{\mathbf{k}}\left(\mathbf{r}_{0}\right)-\varepsilon_{\mathbf{k}}^{(0)}\right]
$$

where $\varepsilon_{\mathbf{k}}\left(\mathbf{r}_{0}\right)$ are the single-electron eigenvalues of the metal substrate in the presence of the adatom at $\mathbf{r}_{0}$ and $\varepsilon_{\mathbf{k}}^{(0)}$ are the corresponding eigenvalues of the free-metal substrate. Furthermore, they also obtained the asymptotic form of $E_{\mathrm{vdW}}(\mathbf{r})$ in the nonoverlap region: They showed ${ }^{27}$ that when the metal substrate is approximated within the jellium model the correlation potential takes the form given by Eq. (5).

The evaluation of the right-hand side of Eq. (12) is a very difficult calculational job. Harris and Liebsch ${ }^{37}$ (HL), avoiding the scattering approach of Zaremba and 
Kohn, ${ }^{31}$ introduced the pseudopotential approach in order to evaluate it in the case of helium atoms interacting with a metal surface. According to the theory of HL, $\varepsilon_{\mathbf{k}}\left(\mathbf{r}_{0}\right)$ can be obtained within the first-order term of the pseudopotential associated with the presence of the adatom $(\mathrm{He}$ atom) at the metal surface. In a recent paper, one of us ${ }^{17}$ has shown that, in the case of the $\mathrm{He}$ atom interacting with metal surfaces, Eq. (12) reduces to Eq. (11) and $\alpha_{\text {eff }}$ can be expressed in terms of quantities which characterize both the metal substrate and the $\mathrm{He}$ atom.

The pseudopotential approach ${ }^{37-39}$ leads to an equation similar to Eq. (11) and has the advantage of being nonlocal. However, the calculated values for $\alpha_{\text {eff }}$ (of the order of $\left.1000 \mathrm{eV} a_{0}^{3}\right)$ are much larger than the values (200 $\mathrm{eV} a_{0}^{3}$ ) found by other methods ${ }^{34}$ which employ the localdensity approximation (LDA) to the exchange and correlation potentials. ${ }^{32-34}$ Takada and $\mathrm{Kohn},{ }^{40}$ reformulating the theory of Zaremba and Kohn ${ }^{31}$ in terms of the $S$ matrix for evanescent wave functions, found $\alpha_{\mathrm{eff}}=490 \mathrm{eV} a_{0}^{3}$ for the zeroth-order Fourier component (smooth part) of $E_{\text {rep }}(\mathbf{r})$. Intermediate values for $\alpha_{\text {eff }}\left(362-383 \mathrm{eV} a_{0}^{3} \mathrm{de}-\right.$ pending on the helium-metal distance) were also found by Batra $^{41}$ and by Batra et al. ${ }^{42}$ who employed a cluster model with Hartree-Fock wave functions. A distancedependent $\alpha_{\text {eff }}$ was reported recently by Rao et al., ${ }^{43}$ who also noted that the Hartree-Fock values of $\alpha_{\text {eff }}$ are slightly changed by including correlation effects. Finally, Andriotis $^{17}$ has shown that, within the first-order perturbation term of the helium pseudopotential, $\alpha_{\text {eff }}$ can be expressed as a functional of the free-electron density $\rho(z)$ and $\alpha_{\text {eff }}$ is a slightly varying function of $\rho(z)$ (see Fig. 1 of Ref. 17).

As proposed by Puska et al., ${ }^{33}$ approximate experimental information for the value of $\alpha_{\text {eff }}$ can be obtained from the values of the scattering length of the electrons scattered by a helium atom. ${ }^{33,44}$ It is interesting that the "experimental" values thus obtained seem to agree better with the results of the methods which employ the LDA.

Two reasons may be responsible for the observed discrepancy in the two theoretical approaches. First is the omission of the correlation contribution to the metaladatom interaction by the pseudopotential method, and second is the fact that the approximation of $E_{\text {rep }}\left(\mathbf{r}_{0}\right)$ by the first-order term of the perturbation series in the helium pseudopotential is not adequate. The latter explanation is supported by the fact that the second-order contribution of the He pseudopotential to $E_{\text {rep }}\left(\mathbf{r}_{0}\right)$ is quite important, even at intermediate values of the overlap integrals. ${ }^{38}$ To these, one can add the findings of Karicorpi et al. ${ }^{45}$ according to which the LDA underestimates the repulsive interaction between the $\mathrm{He}$ atom and a metal surface.

In the present work we attempt to eliminate one of the two reasons which seem responsible for the stated discrepancy. This is achieved by approximating the many-body correlation term of the metal-adatom interaction by its long-range, single-electron expression, i.e., the image potential. This leads to the expression for $V_{\text {corr }}(\mathbf{r})$ given by Eq. (4), which is taken as an external field acting on the adatom and is explicitly incorporated within the $\mathrm{CHF}$ solution of the adatom. The limits of validity of Eq.
(4) and its possible corrections have been already discussed. Here we add that this is an adequate approximation as long as we confine ourselves to metal-adatom distances which involve small overlaps between the adatom and the substrate electron orbitals.

Our approach to $E(\mathbf{r})$ utilizes the assumption of the first-order pseudopotential theory, i.e., that the singleelectron wave functions $\left|\mathbf{k}_{0}\right\rangle$ of the unperturbed metal substrate undergo the following change in the presence of the adatom:

$$
\left|\mathbf{k}_{0}\right\rangle \rightarrow|\mathbf{k}\rangle=\left|\mathbf{k}_{0}\right\rangle-\sum_{a} S_{a \mathbf{k}_{0}}|a\rangle
$$

where $|\mathbf{k}\rangle$ and $|a\rangle$ stand for $\psi_{\mathbf{k}}(\mathbf{r})$ and $\psi_{a}(\mathbf{r})$, respectively, and

$$
S_{a \mathbf{k}_{0}}=\left\langle a \mid \mathbf{k}_{0}\right\rangle \text {. }
$$

Under the assumption implied by Eq. (13), the right-hand side (rhs) of Eq. (12) takes the form ${ }^{16,17}$

$$
E\left(z_{0}\right)=E^{\mathrm{CHF}}\left(z_{0}\right)-E_{\text {free }}^{\mathrm{HF}}-A V_{\text {Coulomb }}\left(z_{0}\right)+E_{\text {rep }}\left(z_{0}\right),
$$

where

$$
E_{\mathrm{rep}}\left(z_{0}\right)=\sum_{a, \mathbf{k}}\left[\varepsilon_{\mathrm{k}}^{(0)}-\varepsilon_{a}-V_{\mathrm{eff}}\left(z_{0}\right)\right]\left|S_{a k_{0}}\left(z_{0}\right)\right|^{2} .
$$

$A$ denotes the atomic number of the adatom, and $E^{\mathrm{CHF}}\left(z_{0}\right)$ and $E_{\text {free }}^{\mathrm{HF}}$ denote the total Hartree-Fock energies of the perturbed (by the substrate) and unperturbed adatoms, respectively. It is noted that $E^{\mathrm{CHF}}\left(z_{0}\right)$ may or may not include the correlation term $V_{\text {corr }}(\mathbf{r})$. In the latter case the rhs of Eq. (15) will include the extra potential terms $E_{\mathrm{vdW}}\left(z_{0}\right)$ given by Eq. (5).

The first three terms of the rhs of Eq. (15) describe the contribution of $E\left(z_{0}\right)$, which comes from the exchange interactions between the metal and the adatom. This may easily be seen by noting that ${ }^{16}$

$$
\begin{aligned}
E^{\mathrm{CHF}}\left(z_{0}\right)-E_{\mathrm{free}}^{\mathrm{HF}} & \sim \sum_{a} \delta \varepsilon_{a} \\
& \sim A V_{\text {Coulomb }}\left(z_{0}\right)+\sum_{a}\left\langle a\left|V_{x}\right| a\right\rangle .
\end{aligned}
$$

The last term on the rhs of Eq. (15) is the contribution to $E\left(z_{0}\right)$ that comes from the perturbation which the singleelectron orbitals of the substrate feel in the presence of the adatom. $E_{\text {rep }}\left(z_{0}\right)$ is the dominant interaction term and is repulsive. It is noted that our Eqs. (12)-(16) constitute another proof ${ }^{16}$ of the result initially obtained by Harris and Liebsch, ${ }^{37}$ who treated the $\mathrm{He}$ pseudopotential as a perturbation to first order acting on the metal states.

The second-order terms in the $\mathrm{He}$ pseudopotential proved $^{38}$ to be a significant factor of the first-order ones. Also, it can be easily shown that if self-consistency is required with respect to the $\mathrm{He}$ pseudopotential, $E_{\text {rep }}\left(z_{0}\right)$ will be augmented by extra terms which are proportional to $\left|S_{a k}\right|^{4}$ (as the second-order terms in the He pseudopotential). Thus, self-consistency effects are expected to be of relative significance in the calculation of $E_{\text {rep }}\left(z_{0}\right)$. 
In the next section we present numerical results based on the Eqs. (12)-16). The effects of self-consistency, as well as of the approximation described by Eq. (13), will be discussed elsewhere.

\section{NUMERICAL RESULTS}

In Figs. 1 and 2 we present the numerical results, obtained according to Eqs. (15) and (16), for the interaction energy $E(z)$ between a helium atom and the semi-infinite metals $\mathrm{Na}(001)$ and $\mathrm{Al}(001)$, respectively. Huzinaga's ${ }^{46} 7 \mathrm{~s}$ set of GTO's was used to describe the $1 s$ state of the helium atom. The metal surface is taken to be the first lattice plane of the crystal near the vacuum region and assumed to be located at $z=0$. The results shown by open circles in Figs. 1 and 2 correspond to $E(z)$, which does not include correlation (van der Waals) effects. Those shown by triangles correspond to $E(z)$, which includes correlation effects according to Eq. (4). The difference between these two results for $E(z)$ is termed the image-correlation potential, $E_{\text {im }}(z)$. From Figs. 1 and 2 it is observed that in the case for which correlation effects are included the interaction energy exhibits a potential-well minimum of about $4 \mathrm{meV}$ for $\mathrm{Na}(001)$ and $7 \mathrm{meV}$ for $\mathrm{Al}(001)$. These values are in agreement with published values of other calculations. ${ }^{31,37,47}$ However, the location of the potential wells we found appears at larger metal-adatom separa-

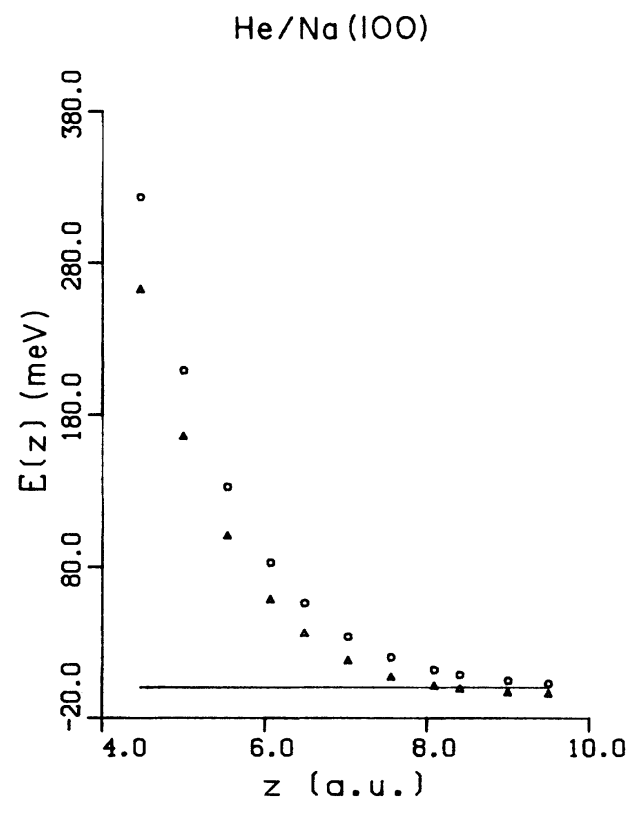

FIG. 1. The interaction $E(z)$ between a helium atom and the $\mathrm{Na}(001)$ semi-infinite metal as given by Eqs. (15) and (16) and within the coupled Hartree-Fock approximation. The results shown by open circles refer to the case of $E(z)$ which does not include the correlation (van der Waals) contribution to $E(z)$. The triangles exhibit the variation of $E(z)$ when correlation effects are included according to Eq. (4) with $z_{\text {im }}=0.0$. The metal surface is taken to be the lattice plane nearest to the vacuum region and is located at $z=0.0$.

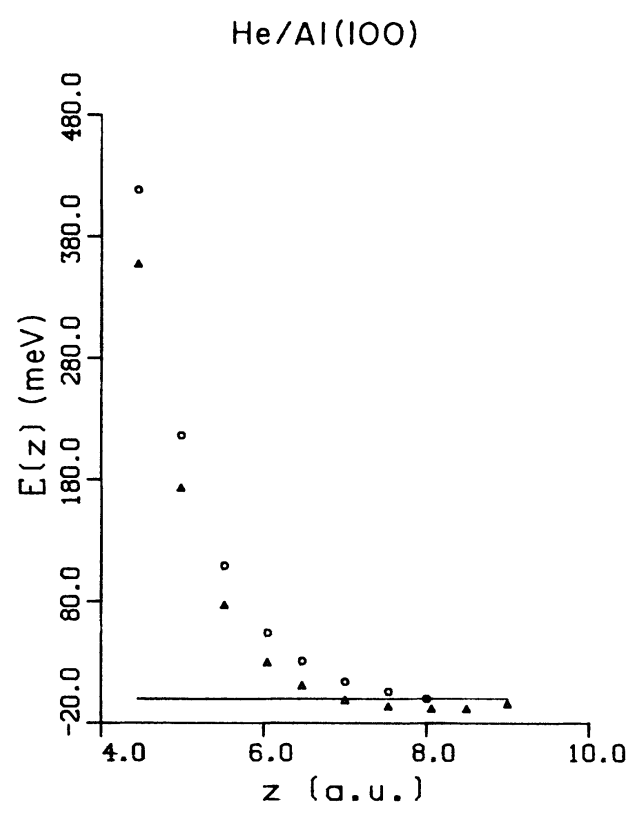

FIG. 2. The interaction energy $E(z)$ between $\mathrm{He}$ and $\mathrm{Al}(001)$ (see caption of Fig. 1).

tions.

A better insight into the interaction-energy curves can be achieved by examining the functional relationship $E(z)=f[\rho(z)]$. When $E(z)$ is plotted against the freeelectron density $\rho(z)$ of the metal, it is found that, in the absence of image-correlation effects, the graphs exhibit a linear relationship between $E(z)$ and $\rho(z)$. In particular, we found that $\alpha_{\text {eff }}=(980 \mathrm{eV}) a_{0}^{3}$ in the case of $\mathrm{Na}(001)$ and for $\rho(z)>2 \times 10^{-5}$ a.u. For $\rho(z)<2 \times 10^{-5}$ a.u. the function $E(z)=E(\rho(z))$ exhibits a variation which reflects the functional relationship $V_{\text {eff }}(z)=V_{\text {eff }}[\rho(z)]$. In the case of $\mathrm{Al}(001)$ a different picture is observed. Initially, $\alpha_{\text {eff }}$ is about $(1100 \mathrm{eV}) a_{0}^{3}$, but for $\rho(z)>1.4 \times 10^{-4}$ a.u., $\alpha_{\text {eff }}$ starts dropping to much smaller values [of about $(650$ $\left.\mathrm{eV}) a_{0}^{3}\right]$. It seems that the dependence of $\alpha_{\text {eff }}$ on the functional $V_{\text {eff }}(z)=V_{\text {eff }}[\rho(z)]$ in the LDA is more pronounced in the case of $\mathrm{Al}(001)$. These observations are in agreement with the relationship $\alpha_{\text {eff }}(z)=\alpha_{\text {eff }}[\rho(z)]$ proposed recently by Andriotis. ${ }^{17,36}$

Figure 3 shows the variation of $\alpha_{\mathrm{eff}}(z)$ as a function of the distance between the helium atom and the $\mathrm{Na}(001)$ metal surface. Triangles denote the results of the present numerical approach. The solid line exhibits the analytic approximation given by Eq. (17) of Ref. 17. The dashed curve exhibits the same analytic expression, ${ }^{17}$ but at a different level of approximation obtained by evaluating the overlap integrals where $\xi_{q}(z)$ is replaced by $\xi_{q}\left(z_{0}\right)$ (its value at the position of the helium atom) over the whole integration range.

The large difference (up to 50\%) found in these two sets of results indicates the sensitivity of the calculations to the approximation employed for the computation of the analytic expression. It seems that both approxima- 


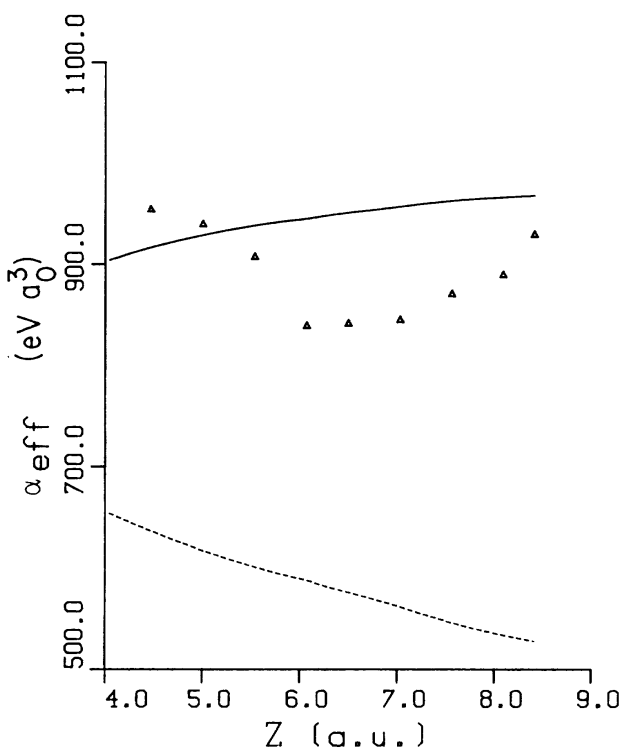

FIG. 3. Dependence of $\alpha_{\text {eff }}$ on the distance between the metal surface, located at $z=0.0$, and the helium atom. Triangles denote the results of the present work. Solid and dashed curves correspond to the analytic expression of $\alpha_{\text {eff }}$ (given in Ref. 17) in two different levels of approximation (see text).

tions are inadequate as they tacitly imply that $\xi_{q}(z)$ and $\xi_{q}^{\prime \prime}(z)$ have the same value over the whole $z$ interval $(-\infty,+\infty)$. For this reason, in the present calculations we avoided the analytic approximation and we used the exact single-electron wave functions obtained numerical$1 \mathrm{y} .^{22}$

The values for $\alpha_{\text {eff }}$ which were found are of the same order of magnitude as the ones found by other workers who employed the same method. ${ }^{37}$ Although these are much larger than the results of other calculations, our results approximately exhibit the same $z$ dependence found by Rao et $a l .{ }^{43}$ and by Batra et al. ${ }^{42}$ Thus, the present work supports previous findings ${ }^{42,43}$ which indicated that $\alpha_{\text {eff }}$ is a functional of the free-electron density $\rho(z)$ of the metal probed by the helium atom. ${ }^{17}$ For this reason, rather than plotting $\alpha_{\text {eff }}$ versus $z$, a more meaningful graph is the one exhibiting the relation between $E(z)$ and $\rho(z)$. From this graph one can, in principle, obtain the approximate value of $\alpha_{\text {eff }}$ over a limited region of $\rho(z)$ values.

Our first computations used values for the parameter $z_{\text {im }}$ given by Lang and $\mathrm{Kohn}^{26}$ (increased by half the interplane distance between the lattice planes of the substrate which are parallel to the surface). However, the image-correlation potential $E_{\mathrm{im}}(z)$ obtained with these values was found to be much stronger than that given by Eq. (5). Thus, in order to have a reasonable value for $z_{\text {im }}$ which we could use along with our formalism, we have proceeded as follows: We used the system $\mathrm{He} / \mathrm{Na}(001)$ as a test system and by trial and error we found the value of $z_{\text {im }}$ which when used according to the present formalism results in an image-correlation potential $E_{\mathrm{im}}(z)$ which coincides approximately with the correlation potential $E_{\mathrm{vdW}}(z)$ given by Eq. (5). As for the parameters of $E_{\mathrm{vdW}}(z)$, i.e., the constants $z_{\mathrm{ZK}}$ and $C_{\mathrm{ZK}}$, we tried those given by Zaremba and $\mathrm{Kohn}^{27}$ as well as those given by Persson and Zaremba, ${ }^{28}$ taking care to increase the given $z_{\mathrm{ZK}}$ values by half the interplane distance of the parallelto-the-surface lattice planes. It was then found that a good agreement between $E_{\mathrm{im}}(z)$ and $E_{\mathrm{vdW}}(z)$ can be obtained by taking $z_{\mathrm{im}}=0.0$. Similarly, $z_{\mathrm{im}}=0.0$ was also found in the case of the $\mathrm{Al}(001)$ substrate.

Having fixed the value of $z_{\text {im }}$ for the test systems and assuming this to be a property of the substrate only, we used the specified value of $z_{\text {im }}$ for adatoms other than the He atoms.

In Fig. 4 we present results for the interaction energy $E(z)$ between an argon atom in its ground state and the $\mathrm{Na}(001)$ semi-infinite metal. We have used the Gaussian basis set of Roos and Siegbahn ${ }^{48}$ to describe the orbitals of the ground state of the argon atom. For the excited argon we had to enlarge this basis set by adding two diffuse GTO's whose exponents were found by energy optimization. ${ }^{29}$ From Fig. 4 it is observed that the interactionenergy curve which includes correlation effects according to Eq. (4) exhibits a potential well of about $60 \mathrm{meV}$. This value is in good agreement with the well of $70 \mathrm{meV}$ found by $\mathrm{Lang}^{49}$ for argon onto silver, the latter approximated by the jellium model. .

Figure 5 exhibits the relative stability between two configurations of the argon adatom onto the $\mathrm{Na}(001)$ semiinfinite metal. In particular, $\Delta E(z)$ is the difference between the interaction energy of the ionic configuration of argon, namely $\mathrm{Ar}^{+}:\left(K L 3 s^{2} 3 p^{52} P^{o}\right)$, and the interaction

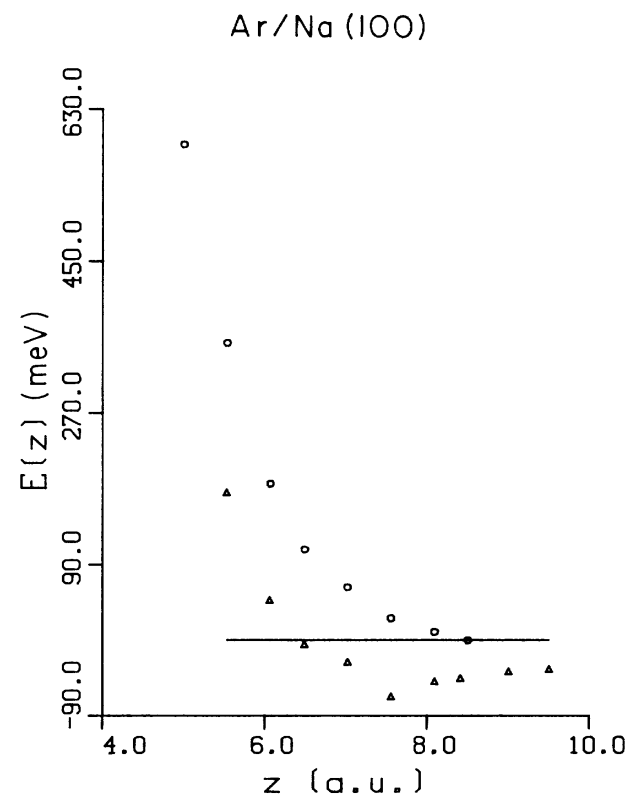

FIG. 4. The interaction energy $E(z)$ between $\mathrm{Ar}$ (in its ground state) with the $\mathrm{Na}(001)$ semi-infinite metal (see caption of Fig. 1). 


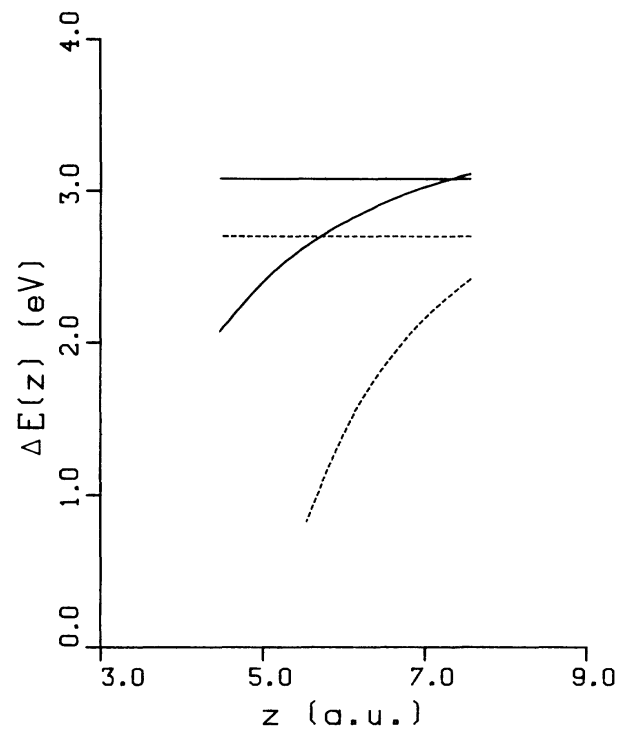

FIG. 5. The energy differences $\Delta E(z)$ given by Eq. (18) of the text as a function of the distance $z$ from the metal surface for argon interacting with $\mathrm{Na}(001)$ semi-infinite metal. The horizontal solid (dashed) line is drawn at the calculated (experimental) work function of the substrate.

energy of the excited argon, $\operatorname{Ar}^{*}:\left(K L 3 s^{2} 3 p^{5} 4 s^{3} P^{o}\right)$, i.e.,

$$
\begin{aligned}
\Delta E(z)= & E_{\mathrm{Ar}^{+}}^{\mathrm{CHF}}(z)-E_{\mathrm{Ar}^{*}}^{\mathrm{CHF}}(z)+E_{\mathrm{rep}}\left(\mathrm{Ar}^{+} ; z\right) \\
& -E_{\mathrm{rep}}\left(\mathrm{Ar}^{*} ; z\right)+V_{\mathrm{im}}\left(z ; z_{\mathrm{im}}\right)
\end{aligned}
$$

where $E_{\mathrm{Ar}^{+}}^{\mathrm{CHF}}\left(E_{\mathrm{Ar}^{*}}^{\mathrm{CHF}}\right)$ is the total energy of the interacting adatom $\mathrm{Ar}^{+}\left(\mathrm{Ar}^{*}\right)$ within the present approach; $E_{\text {rep }}\left(\mathrm{Ar}^{+} ; z\right)$ and $E_{\text {rep }}\left(\mathrm{Ar}^{*} ; z\right)$ are given by Eq. (16) and refer to $\mathrm{Ar}^{+}$and $\mathrm{Ar}^{*}$, respectively; finally, $V_{\mathrm{im}}\left(z ; z_{\mathrm{im}}\right)$ is the image potential between the positive argon ion and the metal in terms of the position $z_{\text {im }}$ of the image plane, i.e.,

$$
V_{\mathrm{im}}\left(z ; z_{\mathrm{im}}\right)=-\frac{1}{4\left(z-z_{\mathrm{im}}\right)} .
$$

Two approximations for $V_{\mathrm{im}}\left(z ; z_{\mathrm{im}}\right)$ are shown in Fig. 5 . The one shown with the solid line refers to the approximation $z_{\mathrm{im}}=0.0$; that shown by the dashed line refers to $z_{\text {im }}=3.3$ a.u., which is the value given by Lang and $\mathrm{Kohn}^{26}$ increased by half the interplane distance between the (001) lattice planes of the sodium crystal. The horizontal solid (dashed) line in Fig. 5 denotes the theoreti$\mathrm{cal}^{22}$ (experimental ${ }^{50}$ ) value of the work function of the $\mathrm{Na}(001)$ semi-infinite metal. According to Fig. 5 and assuming that the electron which is transferred from the adatom to the substrate takes the energy of the Fermi level of the metal (given by the negative of the work function $\Phi)$, it is expected that the excited physisorbed argon atom will exhibit its ionic configuration, as this state is energetically more stable than the excited state $\operatorname{Ar}^{*}[\Delta E(z)<\Phi]$.

Additional support for this picture comes from the known results of alkali-atom-metal interactions: $\mathrm{Ar}^{*}$ has an outer shell similar to that of the potassium atom. When $\mathrm{K}$ interacts with the $\mathrm{Na}(001)$ metal, it is known to lose its electron to $\mathrm{Na}(001)$ metal due to the difference in the electropositivities between $\mathrm{Na}$ and $\mathrm{K}$.

On the other hand, the results of Fig. 5 are the opposite of those expected by using the $\Phi-I^{*}$ criterion $^{13}$ (as $\Phi_{\mathrm{Na}}=2.7 \mathrm{eV}<I_{\mathrm{Ar}}^{*}=4.14 \mathrm{eV}$ ).

Similar trends were found ${ }^{51}$ for $\mathrm{Li} / \mathrm{Al}(001)$. On the other hand, for $\mathrm{Li} / \mathrm{Na}(001)$ the same method predicts ${ }^{51}$ that the neutral-Li-atom configuration is energetically more favored than the ionic $\mathrm{Li}^{+}\left(1 s^{2}\right)$ state.

In summary, it can be said that Eq. (18) offers an extension of the $\Phi-I^{*}$ criterion. ${ }^{51}$ The advantages of the criterion implied by Eq. (18) is that it includes significant many-body interaction terms in the metal-adatom-adion interaction which are ignored by the $\Phi-I^{*}$ criterion. At long metal-adatom separations, Eq. (18) reduces to the $\Phi$ $I^{*}$ criterion as $\Delta E(z)$ becomes equal to $I^{*}$.

Flynn and co-workers, ${ }^{11,15}$ in order to test the stability of the excited $\left(\mathrm{Ar}^{*}\right)$ or the ionic $\left(\mathrm{Ar}^{+}\right)$configuration onto $\mathrm{Al}(001)$, checked if the difference

$$
\Delta E_{1}=I^{*}-\phi+\varepsilon-\Delta
$$

is smaller or greater than zero. In Eq. (20), $\varepsilon$ refers to the cohesive energy of $\mathrm{K}$ and $\Delta$ is the image potential calculated at a distance equal to the atomic radius of argon $(\Delta=1.91 \mathrm{eV}$ and $\varepsilon=0.93 \mathrm{eV})$. In this way they concluded that $\mathrm{Ar}^{*}$ is more stable on $\mathrm{Al}(001)$ than $\mathrm{Ar}^{+}$, in agreement with the experiment.

Although some ambiguities remain concerning the ap-

TABLE I. The ionization potentials $I$ and $I^{*}$ of the ground and the first excited state of the free argon atom, its excitation energy for the transition $3 p^{6} \rightarrow 3 p^{5} 4 s$ and the work functions $\Phi_{\mathrm{Na}(001)}$ and $\Phi_{\mathrm{Al}(001)}$ found in the present calculations are compared with the corresponding experimental values or the exact numerical Hartree-Fock ones. All energies are given in $\mathrm{eV}$.

\begin{tabular}{lccc}
\hline \hline & This work & Expt. & Exact $\mathrm{HF}^{\mathrm{d}}$ \\
\hline $\begin{array}{l}\text { Ionization } \\
\text { potential } I \\
\quad 3 p^{6} \rightarrow\left(3 p^{5}\right)^{+}\end{array}$ & 14.71 & $15.76^{\mathrm{a}}$ & 14.77 \\
$\begin{array}{l}\text { Ionization } \\
\text { potential } I^{*}\end{array}$ & & & \\
$\quad\left({ }^{3} P\right) 3 p^{5} 4 s \rightarrow\left(3 p^{5}\right)^{+}$ & & & \\
$\quad \begin{array}{l}\text { Excitation } \\
\quad \text { energy } \hbar \omega_{a}\end{array}$ & 10.80 & $11.58^{\mathrm{a}}$ & 10.90 \\
$\quad 3 p^{6} \rightarrow 3 p^{5} 4 s\left({ }^{3} P\right)$ & & & \\
$\quad \begin{array}{l}\mathrm{a}(001) \\
\Phi_{\mathrm{Al}(001)}\end{array}$ & 3.08 & $2.70^{\mathrm{b}}$ & \\
\hline \hline
\end{tabular}

${ }^{\mathrm{a}}$ C. E. Moore, Atomic Energy Levels (U.S. GPO, Washington, D.C., 1949), Vol. I.

${ }^{\text {b } R e f e r e n c e ~} 49$ (polycrystalline sample).

'J. Hölzl, in Solid Surface Physics, Vol. 85 of Springer Tracts in Physics (Springer-Verlag, Berlin, 1979).

${ }^{\mathrm{d}} \mathrm{G}$. Aspromallis (private communication). 
plicability of Eq. (20), this equation implies an extension of the original $\Phi-I^{*}$ criterion which is analogous to Eq. (18). Our main question about Eq. (20) is that $\Delta E_{1}$ is distance independent. Besides that, the use of the cohesiveenergy term is a very poor approximation at long metaladatom separations. On the other hand, Eq. (18) does not have these drawbacks, while it takes into account the changes that the substrate electrons undergo in the presence of the adatom. Equation (20) applied to the case of $\mathrm{Ar} / \mathrm{Na}(001)$ gives $\Delta E_{1}=0.44 \mathrm{eV}$ and therefore predicts that the ionic configuration, $\mathrm{Ar}^{+}$, is more stable on $\mathrm{Na}(001)$ than the excited one, $\mathrm{Ar}^{*}$. Thus, the prediction of Eq. (20) about the stable excited configuration of argon on $\mathrm{Na}(001)$ coincides with that given by Eq. (18) over most of the interaction region, i.e., for $z \leq 7.0$ a.u.

In Table I we give numerical results for different physical quantities in order to indicate the degree of accuracy achieved in our calculations. It is worth mentioning that our analytic Hartree-Fock (HF) results are in agreement with the numerical HF results obtained using Froese Fischer's code. ${ }^{52}$

Finally, it is assumed that the intra-atomic correlation effects do not change upon going from a free to a physisorbed and/or chemisorbed atom and therefore they can be obtained from calculations on free atoms. Thus Eq.
(18) can be completed by adding to the rhs a distanceindependent term which corrects for the intra-adsorbate changes of the atomic correlation energy associated with the configurational changes which may take place on the adsorbate. ${ }^{3}$

\section{CONCLUSIONS}

Based on the concept of a "dressed atom" and the calculational scheme of the coupled Hartree-Fock approximation, we present an approach to a series of problems of solid-state physics which exhibit strong characteristics of atomic nature. This approach allows the use of sophisticated numerical and many-body techniques of atomic physics for the accurate description of atom-localized interelectronic interactions. This holds for the ground as well as for the excited states of the adsorbed or embedded atoms.

In this work we have addressed the problem of physisorption on metallic surfaces. The lattice structure of the surface as well as the metal-adatom interactions beyond the simple image-potential approximation were taken into account. The theory is suitable for the calculation of properties such as transition probabilities and inner-electron binding energies.
${ }^{1}$ D. R. Beck and C. A. Nicolaides, in Excited States in Quantum Chemistry, edited by C. A. Nicolaides and D. R. Beck (Reidel, Dordrecht, 1978), p. 329.

${ }^{2}$ D. R. Beck and C. A. Nicolaides, Int. J. Quant. Chem. S 14, 323 (1980).

${ }^{3}$ C. A. Nicolaides, Chem. Phys. Lett. 19, 69 (1973).

${ }^{4}$ C. A. Nicolaides and A. N. Andriotis, Solid State Commun. 44, 99 (1982).

${ }^{5}$ C. A. Nicolaides and A. N. Andriotis, Int. J. Quant. Chem. 23, 561 (1983).

${ }^{6}$ A. N. Andriotis and C. A. Nicolaides, Solid State Commun. 51, 251 (1984).

${ }^{7}$ H. D. Cohen and C. C. J. Roothaan, J. Chem. Phys. 43, 534 (1965).

${ }^{8}$ R. McWeeny and G. Diercksen, J. Chem. Phys. 49, 4852 (1968).

${ }^{9}$ C. A. Nicolaides, M. Papadopoulos, and J. Waite, Theor. Chim. Acta 61, 427 (1982).

${ }^{10}$ C. A. Nicolaides, A. Zdetsis, and A. N. Andriotis, Solid State Commun. 50, 857 (1984).

${ }^{11}$ D. Gibbs, J. E. Cunningham, and C. P. Flynn, Phys. Rev. B 29, $5292(1984)$

12J. E. Demuth, Ph. Avouris, and D. Schmeiser, Phys. Rev. Lett. 50, 600 (1983).

${ }^{13}$ C. P. Flynn and Y. C. Chen, Phys. Rev. Lett. 46, 447 (1981).

${ }^{14}$ N. D. Lang, A. R. Williams, F. J. Himpsel, B. Reihl, and D. E. Eastman, Phys. Rev. B 26, 1728 (1982).

15 J. E. Cunningham, D. Gibbs, and C. P. Flynn, Phys. Rev. B 29, 5304 (1984)

${ }^{16}$ A. N. Andriotis, Phys. Rev. B 31, 4003 (1985).

${ }^{17}$ A. N. Andriotis, Phys. Rev. B 33, 1482 (1986).

${ }^{18}$ N. Esbjerg and J. K. Nørskov, Phys. Rev. Lett. 45, 807 (1980); M. J. Stott and E. Zaremba, Phys. Rev. B 22, 1564 (1980); J. Harris and A. Liebsch, J. Phys. C 15, 2275 (1982); M. J. Pus- ka, R. M. Nieminen, and M. Manninen, Phys. Rev. B 24, 3037 (1981); Y. Takada and W. Kohn, Phys. Rev. Lett. 54, 470 (1985); J. Tersoff, ibid. 55, 140 (1985); Y. Takada and W. Kohn, ibid. 55, 141 (1985).

${ }^{19}$ D. R. Beck and C. A. Nicolaides, in Excited States in Quantum Chemistry, Ref. 1, p. 105.

${ }^{20} \mathrm{C}$. A. Nicolaides, in Advanced Theories and Computational Approaches to the Electronic Structure of Molecules, edited by C. E. Dykstra (Reidel, Dordrecht, 1981), p. 161.

${ }^{21}$ A. N. Andriotis, Surf. Sci. 116, 501 (1982).

${ }^{22}$ A. N. Andriotis, Phys. Rev. B 32, 5062 (1985).

${ }^{23}$ P. R. Antoniewitz, Phys. Rev. Lett. 32, 1424 (1974).

${ }^{24}$ A. G. Eguiluz, D. A. Campell, A. A. Maradudin, and R. F. Wallis, Phys. Rev. B 30, 5449 (1984).

${ }^{25}$ A. G. Eguiluz, Phys. Rev. Lett. 51, 1907 (1983).

${ }^{26}$ N. D. Lang and W. Kohn, Phys. Rev. B 7, 3541 (1973).

${ }^{27}$ E. Zaremba and W. Kohn, Phys. Rev. B 13, 2270 (1976).

${ }^{28}$ B. N. J. Persson and E. Zaremba, Phys. Rev. B 30, 5669 (1984).

${ }^{29}$ C. C. J. Roothaan, Rev. Mod. Phys. 23, 69 (1951).

${ }^{30}$ Hunt's GVBONE version modified for this work by the present authors.

${ }^{31}$ E. Zaremba and W. Kohn, Phys. Rev. B 15, 1769 (1977).

${ }^{32}$ M. J. Stott and E. Zaremba, Phys. Rev. B 22, 1564 (1980).

${ }^{33}$ M. J. Puska, R. M. Nieminen, and M. Manninen, Phys. Rev. B 24, 3037 (1981).

${ }^{34}$ M. Manninen, J. K. Nørskov, M. J. Puska, and C. Umrigar, Phys. Rev. B 29, 2314 (1984).

${ }^{35}$ F. Toigo and M. W. Cole, Phys. Rev. B 32, 6989 (1985).

${ }^{36}$ A. N. Andriotis, Solid State Commun. 59, 761 (1986).

37J. Harris and A. Liebsch, J. Phys. C 15, 2275 (1982).

${ }^{38}$ P. Nordlander and J. Harris, J. Phys. C 17, 1141 (1984); P. Nordlander, Surf. Sci. 126, 675 (1983).

${ }^{39}$ S. Ossicini, Phys. Rev. B 33, 873 (1986). 
${ }^{40}$ Y. Takada and W. Kohn, Phys. Rev. Lett. 54, 470 (1985); J. Tersoff, ibid. 55, 140 (1985); Y. Takada and W. Kohn, ibid. 55, 141 (1985); Y. Takada and W. Kohn, Institute for Theoretical Physics (Santa Barbara, CA )Report No. NSFITP-84-128, 1986 (unpublished).

${ }^{41}$ I. P. Batra, Surf. Sci. 148, 1 (1984).

${ }^{42}$ I. P. Batra, P. S. Bagus, and J. A. Barker, Phys. Rev. B 31, 1737 (1985).

${ }^{43}$ B. K. Rao, P. Jena, D. D. Schillady, A. Hintermann, and M. Manninen, Solid State Commun. 55, 725 (1985).

${ }^{44}$ M. W. Cole and F. Toigo, Phys. Rev. B 31, 727 (1985).
${ }^{45}$ M. Karicorpi, M. Manninen, and C. Umrigar, Surf. Sci. 169, 299 (1986).

46S. Huzinaga, J. Chem. Phys. 42, 1293 (1965).

${ }^{47}$ N. D. Lang and J. K. Nørskov, Phys. Rev. B 27, 4612 (1983).

${ }^{48}$ B. Roos and P. Siegbahn, Theor. Chim. Acta (Berlin) 17, 209 (1970).

${ }^{49}$ N. D. Lang, Phys. Rev. Lett. 46, 842 (1981).

${ }^{50}$ N. D. Lang and W. Kohn, Phys. Rev. B 3, 1215 (1971).

${ }^{51}$ A. N. Andriotis (unpublished).

${ }^{52}$ C. Froese Fischer, Comput. Phys. Commun. 14, 145 (1978). 